\title{
Modern therapeutic management of temporomandibular disorders: A review
}

\author{
Horia Lazarescu' ${ }^{1}$ Doriana Agop-Forna², Claudiu Topoliceanu², \\ Agnes Katherine Lackner ${ }^{3}$, Andrei Kozma ${ }^{1,4}$ \\ ${ }^{1}$ National Institute for Recovery, Physical Medicine and Balneoclimatology, Bucharest, Romania \\ ¿University of Medicine and Pharmacy "Grigore T. Popa", Iasi, Romania \\ ${ }^{3}$ University Dental Clinic, Department of Pediatric Dentistry Medical University Vienna, Austria \\ 4"Alessandrescu-Rusescu" National Institute for Mother and Child Health, Bucharest, Romania
}

\begin{abstract}
The effective temporomandibular disorders (TMD) therapeutic management can be performed only if the causal factors are accurately detected. A modern TMD management favourises approach from a biopsychosocial model where active and adaptive type treatments are fundamental. The management of psychological and behavioral factors must be considered and approached simultaneously with physical factors to accelerate the return to normal functioning of TMD patients with chronic pain. The new multimodal approach of TMD consider self-management as the most important part of TMD therapy, with primary focus on the change of the behavior of TMD patients (Greene). This approach include various components as follows: dental treatments (occlusal adjustments, stabilization splints), cognitive behavioral therapy, relaxation techniques, physical therapy (electrotherapy techniques, low-level laser therapy, neuromusculoskeletal techniques, acupuncture), anti-inflammatory medication.

Conclusions. The modern management of TMD must focuses on the implementation of effective therapeutic algorithms based on accurate diagnostic and assessment of clinical and biological indices. TMD treatment implies multidisciplinary intervention of dentists, physicians, physiotherapists and psychologists.
\end{abstract}

Keywords: temporomandibular disorders, therapeutic management, occlusion adjustements

\section{INTRODUCTION}

Temporomandibular disorders (TMD) are associated to signs and symptoms that affect temporomandibular joint (TMJ) and the muscles involved in jaw movement. TMD are considered a major public health due to negative impact on quality of life by pain and functional impairment (1). The chronic TMD pain prolonged over 6 months is highly associated with psychic depression and pain transfer to somatic level (2). TMD may affect the head control and body position, considering the anatomical connections between the stomatognathic system's proprioceptive inputs and nervous structures implicated in posture, when the proprioceptive information of the stomatognathic system is inaccurate (3). A few studies found significantly impaired oral health-related quality of life (OHRQoL) in patients with a specific TMD diagnosis compared to subjects unaffected by TMD $(4,5)$.

An effective TMD therapeutic management can be performed only if the causal factors are accurately detected. The ethiological factors of TMD onset and development are as follows: macro and microtraumas, stress, arthritis, systemic diseases, teeth loss, age, parafunctional habits $(6,7)$. Bruxism is also associated to TMD in young and middle age patients (8). A review found the absence of a disease-specific association between TMD and occlusal disorders, as the occlusal interferences seem to be the result, and not the cause, of TMD (9).

\section{THERAPEUTIC MANAGEMENT}

Considering high prevalence of TMD in young adults (10), various research groups are focused on 
founding of effective techniques and procedures for TMD management.

The research groups highlight the importance of the multimodal and multidisciplinary approach in patients affected by TMD to optimise the clinical outcomes.

A modern TMD management favourises approach from a biopsychosocial model where active and adaptive type treatments are fundamental (11). However, most patients receive unique treatments and only from one point of view (11). In this context, TMD diagnostic and treatment implies multidisciplinary intervention of dentists, physicians, physiotherapists and psychologists, working in specialized units (11).

The new multimodal approach of TMD consider self-management as the most important part of TMD therapy, with primary focus on the change of the behavior of TMD patients (12). This approach include various components as follows: dental treatments, cognitive behavioral therapy, relaxation techniques, physical therapy, anti-inflammatory and antalgic medication (13).

The most common dental treatments on TMD patients are occlusion adjustments and stabilization splints.

The stabilization splints are used as primary treatment or in combined treatment in TMD pathologies $(14,15)$. The stabilization splints can be also used to protect TMJ structures in TMD patients with occlusal TMD sequelae (16). The stabilization splints are also indicated for TMD patients with arthralgia, diagnosed with incipient symptomatic disc displacement without reduction, or patients with risk factors for degenerative processes (hypoplasic condyles, hypermobility, sleep bruxism) (17). The stabilization splints must be used during night or at most two-thirds of the day to avoid occlusal changes (11).

The need for occlusion adjustments is controversial as stomatognathic system has ability to adapt to changes, including teeth eruption and teeth loss (11). The removal of occlusal interferences is especially requested for patients with parafunctions and those with reduced adaptability to adapt to occlusal changes (18).

The management of psychological and behavioral factors must be considered and approached simultaneously with physical factors to accelerate the return to normal functioning of TMD patients with chronic pain (19).

Psychological therapy addressed to stress, depression, fear, anxiety, fear of movement, must be included in the TMD therapeutic management of this group of patients (20). The most recommended technique is progressive muscular relaxation that trigger a neurophysiological response, muscle relaxation and decreases the neuroendocrine reaction to an adverse event (21). To decrease the pain in patients with TMD associated to headache or chronic back, this technique can be associated with externally control by electromyography or a biofeedback system (22).

Physical therapy is considered for patients with impaired mobility, joint sounds, head and neck pain, or chronic back pain associated to TMD. The physical therapy can be performed by techniques as follows: electrotherapy, low-level laser therapy (LLLT), neuromusculoskeletal physical therapy, self-management techniques, and acupuncture (23).

The most common electrotherapy technique used for the improvement of stomatognathic system functions in TMD patients is transcutaneous electrical nerve stimulation (TENS). TENS technique is performed using controlled exposure of electrical current to the surface of skin, aiming to produce hyperactive muscles relaxation and the decrease of the masticatory muscle pain (24). The assessment of pain intensity, pressure pain threshold and electromyography activity in TMD patients treated by TENS technique reported the decrease of facial pain, deep pain sensitivity and the improvement of masticatory muscle EMG activity (masseter and anterior temporalis during habitual chewing, anterior temporalis during maximal voluntary contraction) (25). A review regarding ultra low frequency-transcutaneous electrical nerve stimulation (ULF-TENS) on patients with temporomandibular disorders (TMD) concluded that ULF-TENS supports the management of TMD patients, but its application should be monitored by electromyographic and electrognathographic analysis performed pretreatment and after the finalization of ULFTENS sessions (26). The reviewers of TENS technique consider that randomized studies with proper design are requested to determine the best protocol for utilization of this technique in the management of TMD patients. 
Low-level laser therapy (LLLT) is reported to be effective in reducing the levels of TMD pain. The researchers investigated laser assisted TMD treatment performed between one and 20 sessions, in daily or weekly sessions, using energy density ranged from 0.9 to $105 \mathrm{~J} / \mathrm{cm}^{2}$, and power density ranged from 9.8 to $500 \mathrm{~mW}$. The reviewers of LLLT studies highlight the heterogeneity of the laser parameters and request a consensus regarding the best application protocol for pain relief in patients with $\operatorname{TMD}(27,28)$.

Neuromusculoskeletal physical therapy for patients with TMD uses manual techniques to improve TMJ movement and to decrease masticatory muscles pain, followed by therapeutic exercises that will further optimise the clinical outcome (29). For TMD patients neuromusculoskeletal physiotherapy is recommended not only to the orofacial region but also to the upper cervical region, to reduce pain and improve the stomatognathic system functions (30).

Acupuncture is an alternative therapeutic technique with short-term benefits in pain reduction to patients with myogenic TMD, but without significant results on improvement of TMJ movement (31). The acupuncture applied in the orofacial re-

\section{REFERENCES}

1. Sória TS, Sória GS, Cruz RA, Brew MC, Grossmann E, Bavaresco CS. Effect of Temporomandibular Disorders on Quality of Life for Elderly Individuals. Iran J Public Health. 2018 Oct; 47(10): 1599-1601.

2. Manfredini D, Winocur E, Ahlberg J, Guarda-Nardini L, Lobbezoo F. Psychosocial impairment in temporomandibular disorders patients. RDC/TMD axis II findings from a multicenter study. J Dent. 2010 Oct; 38(10): 765-72.

3. Cuccia A, Caradonna C.The relationship between the stomatognathic system and body posture. Clinics (Sao Paulo). 2009; 64(1):61-6.

4. Reissmann DR, John MT, Schierz O, Wassell RW. Functional and psychosocial impact related to specific temporomandibular disorder diagnoses. J Dent. 2007 Aug; 35(8):643-50.

5. Miettinen O, Lahti S, Sipilä K. Psychosocial aspects of temporomandibular disorders and oral health-related quality-of-life. Acta Odontol Scand. 2012 Jul; 70(4):331-6.

6. Fernandes G, van Selms MK, Gonçalves DA, Lobbezoo F, Camparis $\mathrm{CM}$. Factors associated with temporomandibular disorders pain in adolescents. J Oral Rehabil. 2015 Feb; 42(2):113-9.

7. Häggman-Henrikson B, Rezvani M, List T. Prevalence of whiplash trauma in TMD patients: a systematic review. J Oral Rehabil. 2014 Jan; 41(1):59-68.

8. Blanco Aguilera A, Gonzalez Lopes L, Blanco Aguilera E, De la Hoz Aizpura JL, Rodriguez Torronteras A, Segura Saint-Gerons R, Blanco Hungría A. Relationship between self-reported sleep bruxism and pain in patients with temporomandibular disorders. $J$ Oral Rehabil. 2014 Aug; 41(8): 564-72.

9. Manfredini D, Lombardo L, Siciliani G.Temporomandibular disorders and dental occlusion. A systematic review of association studies: end of an era? J Oral Rehabil. 2017; 44(11):908-923. gion has effects on the peripheral opioid receptors and blocks the local nociceptive input during the therapeutic process (32).

\section{CONCLUSIONS}

The modern management of TMD must focuses on the implementation of effective therapeutic algorithms based on accurate diagnostic and assessment of clinical and biological indices. The selection of the treatment techniques must relate to the degree of stomatoghnatic system dishomeostasy and adaptability of the stomatognathic system to occlusal changes. TMD treatment implies multidisciplinary intervention of dentists, physicians, physiotherapists and psychologists. Modern therapeutic approach of TMD is based on multimodal care including self-management, dental treatments, cognitive behavioral therapy, physical therapy (TENS technique, low-level laser therapy, neuromusculoskeletal therapy, acupuncture), and relaxation techniques.

\section{Acknowledgement}

All authors have same/equal contribution.
10. Wieckiewicz M, Grychowska N, Wojciechowski K, Pelc A, Augustyniak M, Sleboda A, Zietek M. Prevalence and correlation between TMD based on RDC/TMD diagnoses, oral parafunctions and psychoemotional stress in Polish university students. Biomed Res Int. 2014; 2014:472346.

11. Garrigós-Pedrón M, Elizagaray-García I, Domínguez-Gordillo AA, Del-Castillo-Pardo-de-Vera JL, Gil-Martínez A. Temporomandibular disorders: improving outcomes using a multidisciplinary approach. J Multidiscip Healthc. 2019; 12:733-747.

12. Greene CS. Managing the care of patients with temporomandibular disorders: a new guideline for care. J Am Dent Assoc. 2010 Sep; 141(9):1086-8.

13. Story WP, Durham J, Al-Baghdadi M, Steele J, Araujo-Soares V. Self-management in temporomandibular disorders: a systematic review of behavioural components. J Oral Rehabil. 2016 Oct; 43(10):759-70.

14. Costa YM, Porporatti AL, Stuginski-Barbosa J, Bonjardim LR, Conti PC. Additional effect of occlusal splints on the improvement of psychological aspects in temporomandibular disorder subjects: A randomized controlled trial. Arch Oral Biol. 2015 May; 60(5):738-44.

15. Conti PC, de Alencar EN, da Mota Corrêa AS, Lauris JR, Porporatti AL, Costa YM Behavioural changes and occlusal splints are effective in the management of masticatory myofascial pain: a short-term evaluation. J Oral Rehabil. 2012 Oct; 39(10):754-60.

16. Magdaleno F, Ginestal E. Side effects of stabilization occlusal splints: a report of three cases and literature review. Cranio. 2010 Apr; 28(2):128-35.

17. Greene CS, Obrez A. Treating temporomandibular disorders with permanent mandibular repositioning: is it medically necessary? Oral Surg Oral Med Oral Pathol Oral Radiol. 2015; 119(5):489-98. 
18. Michelotti A, Cioffi I, Landino D, Galeone C, Farella M. Effects of experimental occlusal interferences in individuals reporting different levels of wake-time parafunctions. J Orofac Pain. 2012 Summer; 26(3):168-75

19. Alrashdan MS, Alkhader M. Psychological factors in oral mucosal and orofacial pain conditions. Eur J Dent. 2017 Oct-Dec; 11(4):548-552.

20. Gil-Martínez A, Paris-Alemany A, López-de-Uralde-Villanueva I, La Touche R. Management of pain in patients with temporomandibular disorder (TMD): challenges and solutions. J Pain Res. 2018; 11():571-587.

21. Scrivani SJ, Khawaja SN, Bavia PF. Nonsurgical Management of Pediatric Temporomandibular Joint Dysfunction. Oral Maxillofac Surg Clin North Am. 2018; 30(1):35-45.

22. Crider A, Glaros AG, Gevirtz RN. Efficacy of biofeedback-based treatments for temporomandibular disorders. Appl Psychophysiol Biofeedback. 2005 Dec; 30(4):333-45.

23. Paço M, Peleteiro B, Duarte J, Pinho T. The Effectiveness of Physiotherapy in the Management of Temporomandibular Disorders: A Systematic Review and Meta-analysis. J Oral Facial Pain Headache. 2016; 30(3):210-20.

24. Awan KH, Patil S.The Role of Transcutaneous Electrical Nerve Stimulation in the Management of Temporomandibular Joint Disorder. J Contemp Dent Pract. 2015 Dec 1; 16(12):984-6.

25. Ferreira AP, Costa DR, Oliveira Al, Carvalho EA, Conti PC, Costa YM, Bonjardim LR. Short-term transcutaneous electrical nerve stimulation reduces pain and improves the masticatory muscle activity in temporomandibular disorder patients: a randomized controlled trial. J Appl Oral Sci. 2017 Mar-Apr; 25(2):112-120.

26. Chipaila N, Sgolastra F, Spadaro A, Pietropaoli D, Masci C, Cattaneo $R$, Monaco $A$. The effects of ULF-TENS stimulation on gnathology: the state of the art. Cranio. $2014 \mathrm{Apr} ; 32(2): 118-30$.

27. Maia ML, Bonjardim LR, Quintans Jde S, Ribeiro MA, Maia LG, Conti PC.Effect of low-level laser therapy on pain levels in patients with temporomandibular disorders: a systematic review. J Appl Oral Sci. 2012 Nov-Dec; 20(6):594-602.

28. Melis M, Di Giosia M, Zawawi KH. Low level laser therapy for the treatment of temporomandibular disorders: a systematic review of the literature. Cranio. 2012; 30(4):304-12.

29. Armijo-Olivo S, Magee D. Cervical musculoskeletal impairments and temporomandibular disorders. J Oral Maxillofac Res. 2012 Oct-Dez; 3(4): 4 .

30. Armijo-Olivo S, Pitance L, Singh V, Neto F, Thie N, Michelotti A. Effectiveness of Manual Therapy and Therapeutic Exercise for Temporomandibular Disorders: Systematic Review and Meta-Analysis. Phys Ther. 2016 Jan; 96(1):9-25

31. Wu JY, Zhang C, Xu YP, Yu YY, Peng L, Leng WD, Niu YM, Deng $\mathrm{MH}$. Acupuncture therapy in the management of the clinical outcomes for temporomandibular disorders: A PRISMA-compliant meta-analysis. Medicine (Baltimore). 2017 Mar; 96(9):e6064.

32. Goddard G, Karibe H, McNeill C, Villafuerte E. Acupuncture and sham acupuncture reduce muscle pain in myofascial pain patients. J Orofac Pain. 2002 Winter; 16(1):71-6. 